\title{
Fibroblast activation protein and its prognostic significance in correlation with vascular endothelial growth factor in pancreatic adenocarcinoma
}

\author{
DIMITRIOS PATSOURAS ${ }^{1}$, KOSTIS PAPAXOINIS ${ }^{2}$, ALKIVIADIS KOSTAKIS ${ }^{3}$, \\ MICHAEL C. SAFIOLEAS ${ }^{4}$, ANDREAS C. LAZARIS ${ }^{5}$ and POLYXENI NICOLOPOULOU-STAMATI ${ }^{5}$ \\ ${ }^{1}$ Department of GI Surgery, St. Thomas' Hospital, London SE1 7EH, UK; ${ }^{2}$ Gastroenterology Unit, \\ 1st Department of Internal Medicine-Propaedeutic, 'Laiko' General Hospital, Athens University Medical School, Athens GR-11527; \\ ${ }^{3}$ Center of Experimental Surgery, Biomedical Research Foundation, Academy of Athens, Athens GR-11527; \\ ${ }^{4}$ Fourth Propedeutic Department of Surgery, Athens University Medical School, Attikon Hospital of Athens, Athens GR-12462; \\ ${ }^{5} 1$ st Department of Pathology, Athens University Medical School, Athens GR-11527, Greece
}

Received March 6, 2014; Accepted November 20, 2014

DOI: $10.3892 / \mathrm{mmr} .2015 .3259$

\begin{abstract}
Fibroblast activation protein (FAP), a selective protein for tumor stromal fibroblasts, is expressed in $>90 \%$ of human epithelial carcinomas. A characteristic feature of pancreatic cancer is an extensive fibrotic or desmoplastic reaction surrounding the primary tumor. The present study aimed to evaluate the expression levels of FAP and vascular endothelial growth factor (VEGF) and determine their correlation in pancreatic adenocarcinoma. Confocal laser scanning microscopy and conventional immunohistochemical analysis were used to quantify FAP and VEGF expression levels in formalin-fixed and paraffin-embedded tissue biopsies from 46 patients (male, 26; female, 20; mean age, 66 years; age range, 53-80 years) with pancreatic adenocarcinoma stage IIA or IIB. The expression levels of FAP in the neoplastic and adjacent normal tissue were significantly higher in stage IIB patients, compared with stage IIA patients. FAP expression was correlated with positive lymph nodes, resulting in poor prognosis for stage IIB patients. The partial correlation coefficient between FAP and VEGF expression levels was $0.39(\mathrm{P}=0.007)$, and the two factors had an effect on patient survival. Multivariate analysis demonstrated the prognostic superiority of FAP over VEGF, which is considered to be the most consistently reproducible molecular marker with prognostic value in resected pancreatic adenocarcinoma. Due to the limited beneficial effect of current systemic therapies for
\end{abstract}

Correspondence to: Mr. Dimitrios Patsouras, Department of GI Surgery, St. Thomas' Hospital, Westminster Bridge Road, London SE1 7EH, UK

E-mail: d.patsouras@gmail.com

Key words: fibroblast activation protein, pancreatic cancer, vascular endothelial growth factor, immunohistochemistry pancreatic adenocarcinoma, targeting FAP may be a potential therapeutic strategy and requires further investigation.

\section{Introduction}

Pancreatic cancer, principally adenocarcinoma of ductal origin, is the fourth most common cause of cancer-associated mortality in the United States (1). Pancreatic cancer has the worst prognosis among all major human tumor types, with a five-year survival rate of only $5 \%$ when all stages are considered (1). Although surgical resection is considered to be the sole curative therapeutic approach, only $10-30 \%$ of patients have resectable tumors at the time of diagnosis (2). Previous studies reported that the five-year survival rate following curative resection is $10-17 \%$ (3-7). Given the limited beneficial effect of conventional chemotherapy in the treatment of pancreatic cancer, novel targets for the treatment of this disease are required.

Angiogenesis is considered to be one of the most important factors for tumor development and progression. Vascular endothelial growth factor (VEGF) plays an important role in tumor angiogenesis and is significantly correlated with tumor invasion and metastasis (8-10). In addition, VEGF has a central role in the emergence of reactive stroma (11). VEGF can be directly released from cancer cells; however, fibroblasts and inflammatory cells are the predominant source of host-derived VEGF (12). Furthermore, VEGF induces microvascular permeability, leading to extravasation of plasma proteins, including fibrin, which initiates an influx of fibroblasts, and inflammatory and endothelial cells $(11,13,14)$. These cells produce extracellular matrix (ECM) that predominantly consists of fibronectin and type I collagen, which are associated with the initiation of tumor angiogenesis $(11,15,16)$. Pancreatic tumors are not considered to be grossly vascularized and are characterized by a dense stromal reaction that can subsequently promote tumor invasion (17). Notably, the majority of pancreatic cancers display overexpression of angiogenic molecules, including VEGF, which is a key mediator of tumor angiogenesis (18-20). 
A characteristic feature of pancreatic cancer is the presence of an extensive fibrotic or desmoplastic reaction surrounding the primary tumor (17). Fibroblast activation protein (FAP) is a selective protein for tumor stromal fibroblasts. FAP is a $95 \mathrm{kDa}$ cell surface glycoprotein expressed by tumor stromal fibroblasts in $>90 \%$ of human epithelial carcinomas, including breast, lung, colorectal and ovary carcinomas $(21,22)$. Previous studies have demonstrated that FAP exhibits dipeptidyl peptidase and collagenase activity (21-23). A considerable number of studies have presented evidence that support the importance of stroma and fibroblasts in cancer invasion and metastasis $(17,24)$. Cancer cells produce enzymes that degrade basement membranes and the ECM, inducing characteristic cellular and molecular alterations to the supporting stroma (24). Carcinoma-associated fibroblasts (CAFs) that express FAP have been shown to directly stimulate tumor cell proliferation, angiogenesis and metastasis by producing various growth factors, hormones and cytokines $(22,23,26-28)$.

Computer-based image analysis is a valuable tool in pathology for measuring structural parameters concerning all aspects of neoplasia, including proliferation, apoptosis, angiogenesis and carcinogenesis, in an objective and reproducible manner. Confocal laser scanning microscopy (CLSM) is an immunohistochemical detection technique that is able to recognize specific cellular proteins. This technique combines the extreme immunofluorescence sensitivity with improved image resolution by capturing images that are almost free of out-of-focus signals (NEW 1 and 2). CLSM has been successfully applied in quantitative pathology to measure the expression of various cellular components, even in ordinary paraffin-embedded sections (31). However, whether formalin-fixed tissues are prone to non-specific autofluorescence remains unclear (NEW 3). Nevertheless, the use of formalin-fixed tissue in the present study did not appear to raise any technical problems.

The present study used a computer-assisted CLSM method to quantify FAP expression, while standard immunohistochemical analysis was used to assess VEGF expression in a series of human pancreatic adenocarcinomas. The aim of the current study was to investigate the correlation of the results in an attempt to elucidate the role of fibroblasts in the progression of pancreatic cancer. In addition, the impact of FAP expression on the overall survival of the patients was investigated, following a standard chemotherapy regimen with gemcitabine after surgery.

\section{Materials and methods}

Patients and specimens. A total of 46 patients (male, 26; female, 20) with a mean age of 66 years (range, 53-80 years) were selected. The participants of this study were pancreatic adenocarcinoma patients who had undergone curative pancreatic resection during a period of six years (2003-2006) at the 'Laiko' General University Hospital (Athens University Medical School, Athens, Greece). Paraffin-embedded samples of pancreatic adenocarcinoma and normal adjacent pancreatic tissue were researched retrospectively and retrieved from the archives of the Pathology Department of 'Laiko' General University Hospital (Athens University Medical School, Athens, Greece) where they were stored from the time of the operation under appropriate conditions. A pathologist blindly reviewed the slides to ensure that the cases were consistent with pancreatic ductal adenocarcinoma, according to the World Health Organization classification (33). The surgical procedure included standard pancreatoduodenectomy and distal pancreatectomy. Patients with distant metastases detected pre-operatively or receiving any type of cancer treatment prior to the surgery were excluded from the study. All the patients received the same chemotherapy regimen following the surgery. The chemotherapy treatment schedule was: $1,000 \mathrm{mg} /$ $\mathrm{m}^{2}$ gemcitabine intrvenously administered in $30 \mathrm{~min}$ on days $1,8,15$ every 28 days for 6 cycles. Each cycle lasted 28 days and the total duration of treatment was six months. Outcome data were recorded from follow-up consultations with the patients, which were arranged according to each patient's oncologist's recommendations. Contact was maintained with the patients and their families, as well as with the physicians involved in the patients' treatment. The patients surviving until the final follow-up visit or contact were included in the survival analysis. At the time the present study was completed, 16 out of 46 patients were alive. A modified Kloppel grading system (34) was used to establish the differentiation grade of the tumor samples as follows: G1, well-differentiated; G2, moderately-differentiated; G3, poorly-differentiated. Tumor node metastasis staging (35) was performed, according to the criteria of the National Comprehensive Cancer Network. The present study was approved by the Ethics Committee of the National and Kapodistrian University of Athens and the Institutional Review Board (Athens, Greece). Written informed consent was obtained from all patients or their families.

Immunohistochemistry and immunofluorescence. All the biopsy specimens were fixed in formalin solution and processed, according to the routine protocol of the Pathology Laboratory of the Athens University Medical School, from the blocks of tissue retrieved. Paraffin-embedded sections $(4 \mu \mathrm{m})$ were stained with hematoxylin and eosin for histological or immunohistochemical analyses. The tissue sections were then treated with a 1:50 dilution of monoclonal mouse anti-human VEGF antibody (M7273; Dako, Glostrup, Denmark). Detection was performed using the Dako Envision Detection system, Peroxidase/DAB+ (K4065; Dako). The levels of immunostaining were evaluated by examining 10 random high-power fields under x 400 magnification. Specimens expressing $>20 \%$ VEGF were arbitrarily considered to 'highly' express the marker. Each specimen was assessed by two pathologists, who had no access to the clinical information, with the use of a two-headed microscope (E400 Eclipse dual viewing microscope; Nikon Corp., Tokyo, Japan). In the case of disagreement between the observers the specimens were re-evaluated, in order to reach a consensus.

Immunofluorescent analysis was also performed on 4- $\mu \mathrm{m}$ sections of neoplastic and adjacent normal tissue from each patient. The following antibodies were used: Mouse monoclonal anti-human FAP (F11-24) antibody (sc-65398; Santa Cruz Biotechnology, Inc., Dallas, TX, USA), at a dilution of 1:50; and fluorescein isothiocyanate (FITC)-labeled goat anti-mouse polyclonal antibody (sc-2010; Santa Cruz Biotechnology, Inc.), at a dilution of 1:100. The tissue sections were incubated with anti-FAP at room temperature for $60 \mathrm{~min}$ 
Table I. Cox regression analysis of survival assessing the impact of multiple covariates in the model. FAP expression in the neoplastic tissue had a significant effect on survival $(\mathrm{P}=0.003)$, whereas other variables did not affect survival $(\mathrm{P}>0.05)$.

A, Dependent variable: Survival

Overall (score)

\begin{tabular}{lccc}
\cline { 2 - 3 }-2 Log likelihood & $\chi^{2}$-value & Df & P-value \\
\hline 142.134 & 15.458 & 7 & 0.009
\end{tabular}

$\mathrm{B}$, Variables in the equation

$95.0 \%$ CI for $\operatorname{Exp}(\mathrm{B})$

\begin{tabular}{lcccccrrr} 
Variable & B & SE & Wald & DF & P-value & Exp (B) & Lower & Upper \\
\hline Age & 0.041 & 0.029 & 2.003 & 1 & 0.157 & 1.041 & 0.984 & 1.102 \\
Gender & 1.129 & 0.637 & 3.141 & 1 & 0.076 & 3.091 & 0.887 & 10.768 \\
Stage & -0.043 & 0.493 & 0.008 & 1 & 0.930 & 0.958 & 0.364 & 2.517 \\
VEGF & -0.915 & 0.495 & 3.414 & 1 & 0.065 & 0.401 & 0.152 \\
FAP_ca & 0.042 & 0.014 & 8.542 & 1 & 0.003 & 1.043 & 1.014 \\
\hline
\end{tabular}

B, estimated coefficient; SE, standard error of B coefficient; Wald, Wald test; DF, degrees of freedom; Exp(B), exponentiation of B coefficient; FAP_ca, fibroblast activation protein in neoplastic tissue; VEGF, vascular endothelial growth factor; CI, confidence interval.

and with anti-FITC for $60 \mathrm{~min}$ at room temperature in the dark. Normal goat serum was used to block non-specific antigenic sites (sc-2043; Santa Cruz Biotechnology, Inc.). All the samples were maintained at $4^{\circ} \mathrm{C}$ in the dark and observed within $24 \mathrm{~h}$ after staining in order to avoid fluorescence fading.

CLSM. Fluorescent images were obtained using a Bio-Rad MRC-600 confocal laser scanning imaging system (Bio-Rad Laboratories, Inc., Hercules, CA, USA) supplied with an argon ion laser source with a maximum emitting power of $25 \mathrm{~mW}$. The imaging system was coupled to a Nikon Optiphot microscope (Nikon Corporation, Tokyo, Japan) under x40 magnification. Integrated excitation and emission filters at 488 and $515 \mathrm{~nm}$, respectively, were used to detect FITC (excitation peak, $490 \mathrm{~nm}$; emission maximum, $525 \mathrm{~nm}$ ). All the images were acquired using the same system settings and quantified using a previously described standard methodology (31). A unique value of $0-255$ units, corresponding to the FAP staining intensity, was attributed to each case.

Statistical analyses. Statistical analyses were performed using SPSS version 13.0 software for Windows (SPSS Inc., Chicago, IL, USA). McNemar's test was used to assess the difference among VEGF expression in the various disease stages. A $\chi^{2}$ test of independence was used to determine whether there was an association between disease stage and VEGF expression. Independent samples t-test was used to compare the differences in FAP expression in the neoplastic tissue, associated with the disease stage and VEGF expression. The correlation between FAP expression in the neoplastic and adjacent normal tissue was assessed by calculating the partial correlation coefficient, controlling the disease stage and VEGF expression.
The effect of VEGF on the survival rate was assessed using the Kaplan-Meier method, while the effect of FAP expression and covariates on the survival rate were investigated using Cox regression. $\mathrm{P}<0.05$ was considered to indicate a statistically significant difference.

\section{Results}

A total of 46 patients (male, 26; female, 20) with a mean age of 66 years (range, 53-80 years) participated in this study. All the tumor samples investigated were found to be moderately or poorly differentiated, with 22 patients presenting stage IIA $\left(\mathrm{T}_{3} \mathrm{~N}_{0} \mathrm{M}_{0}\right)$ disease and 24 patients presenting stage IIB $\left(\mathrm{T}_{1}, \mathrm{~T}_{2}\right.$ or $\mathrm{T}_{3} \mathrm{~N}_{1} \mathrm{M}_{0}$ ) disease. The median survival time was 11 months (range, 4-32 months). VEGF expression was detected in the neoplastic cells and in a few stroma cells, as determined by cytoplasmic staining. VEGF was found to be highly expressed in 11 out of the 22 patients with stage IIA disease and in 19 out of 24 patients with stage IIB $(\mathrm{P}=0.038)$. FAP expression was predominantly expressed in tumor myofibroblasts and, at a lesser extent, in cancer cells (Fig. 1). FAP expression [mean (standard deviation)] was found to be 36.4 (9) arbitrary units in stage IIA disease with negative lymph nodes and 42.7 (11.1) arbitrary units in stage IIB disease with positive lymph nodes $(\mathrm{P}=0.04)$. In patients presenting high expression levels of VEGF, FAP expression was 42.6 (10.7) arbitrary units, while in the patients with low expression of VEGF, FAP expression was $34.3(8)(\mathrm{P}=0.03)$. By contrast, the expression of FAP in the adjacent normal tissue was 28 (7.9) in the patients with stage IIA disease and 30.5 (10.3) in the patients with stage IIB disease ( $P>0.05$; Fig. 2). Therefore, FAP expression in the neoplastic and adjacent normal tissue was found to be 

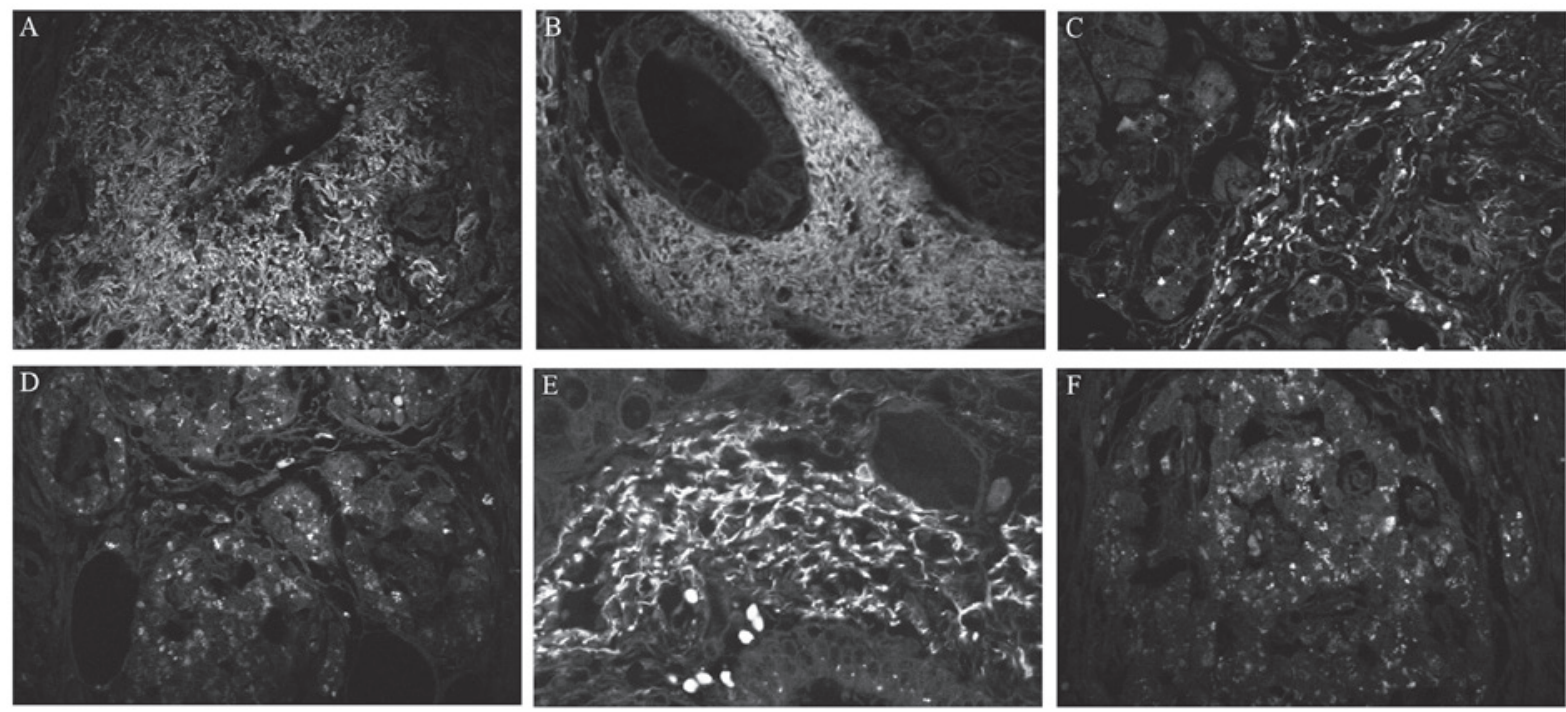

Figure 1. Confocal microscopy images of fibroblast activation protein (FAP) expression in human pancreatic adenocarcinoma tissues. Sections from (A-F) six pancreatic carcinoma cases selected from the 46 studied with extensive fibrotic desmoplastic reaction. FAP was stained using mouse monoclonal anti-human FAP antibody (sc-65398). FAP was predominantly expressed in tumor myofibroblasts and, at a lesser extent, in carcinoma cells (magnification, x400).

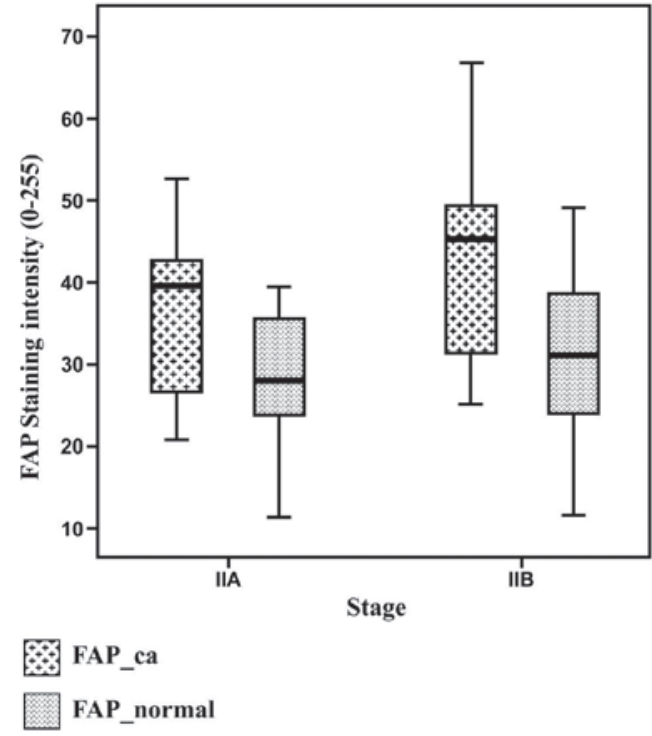

Figure 2. Boxplots displaying fibroblast activation protein (FAP) staining intensity ( $0-255$ arbitrary units) associated with the disease stage. The values [mean (standard deviation)] for stages IIA and IIB were: Neoplastic tissues, 36.4 (9) and 42.7 (11.1), respectively $(\mathrm{P}=0.004)$; and normal adjacent tissues, 28 (7.9) and 30.5 (10.3), respectively $(\mathrm{P}>0.05)$.

significantly correlated (partial correlation coefficient, 0.39; $\mathrm{P}=0.007$ ). This correlation was independent of disease stage and VEGF expression (Fig. 2). The median (standard deviation) survival time for the tumors with low VEGF expression was 23 (2.3) months, as compared with 11 (1.9) months in the tumors with high VEGF expression; therefore, a statistically significant difference was observed between the survival rates $(\mathrm{P}=0.001$; Fig. 3$)$. In the Cox regression model for survival analysis, FAP expression in the neoplastic tissue was shown to significantly affect the survival rate $(\mathrm{P}=0.003)$, while this effect was independent of VEGF expression $(\mathrm{P}=0.065)$, disease stage $(\mathrm{P}=0.930)$, age $(\mathrm{P}=0.157)$ and gender $(\mathrm{P}=0.076$; Table I).
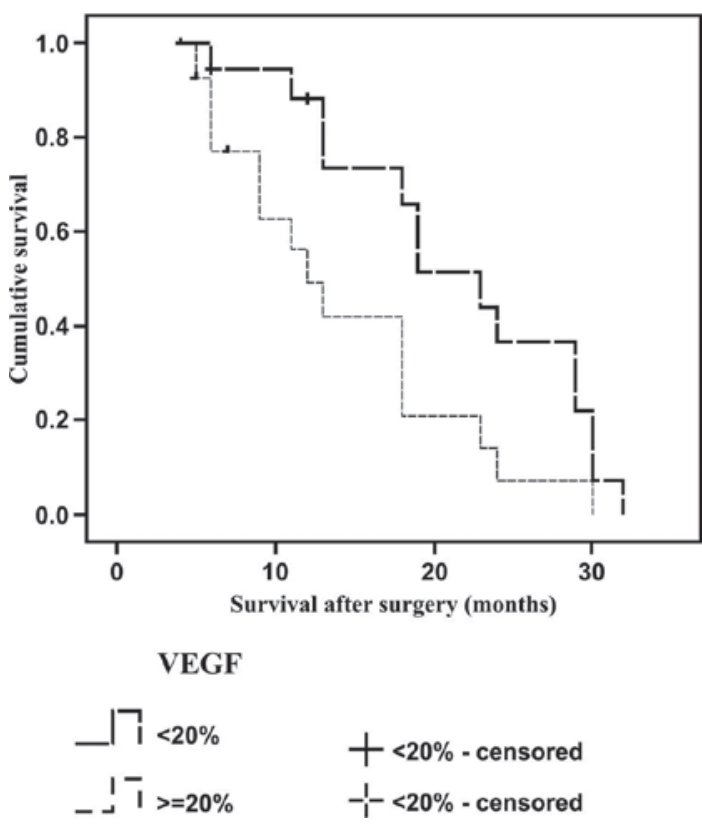

Figure 3. Kaplan-Meier curves for pancreatic adenocarcinoma. The survival rate was significantly reduced in tumors showing high expression of VEGF $(\mathrm{P}=0.001)$.

\section{Discussion}

The results of the present study were concordant with the findings from previous reports, which indicated that FAP may play a significant role in pancreatic adenocarcinoma. FAP has been previously shown to be predominantly expressed in the tumor stroma of epithelial malignancies, while its presence has been associated with epithelial cancer invasion, tumor angiogenesis, and subsequent growth and metastasis $(22,23,26-28,36)$. The present study demonstrated that FAP expression in the neoplastic and adjacent normal tissue was significantly higher in stage IIB patients, when compared with stage IIA patients. In addition, FAP expression was found to be correlated with 
positive lymph nodes and lymphatic vessel invasion, and was therefore associated with poor prognosis in patients with stage IIB disease. Cohen et al (26) demonstrated that high expression of FAP in the surrounding tumor myofibroblasts was associated with positive lymph nodes, reduced time period to recurrence and reduced survival rate. Furthermore, Shi et al (37) demonstrated that FAP was highly expressed in carcinoma cells, as well as in the fibroblasts of pancreatic adenocarcinoma tissues. The authors also identified that patients with a high FAP expression in carcinoma cells had a significantly reduced overall survival rate compared with the patients with low FAP expression, while a multivariate analysis indicated that high expression of FAP was an independent predictor of overall survival. In the present study, a survival analysis was conducted using the Cox regression model, and the expression of FAP in the neoplastic tissues was found to significantly affect survival; however, this effect was independent from VEGF expression, disease stage, age and gender.

In the present study, the survival rate was considerably reduced in patients with higher expression levels of VEGF. This result was concordant with a recent meta-analysis, which suggested that VEGF represents the most consistently reproducible immunohistochemical marker with prognostic value in resected pancreatic adenocarcinoma (38). Furthermore, the results of the present study demonstrated that a higher VEGF expression in pancreatic cancer is correlated with a higher expression of FAP in the same tissue. To the best of our knowledge, this is the first study to demonstrate a correlation between FAP and VEGF expression levels and evaluate their association with clinical outcomes in pancreatic adenocarcinoma.

Currently, anti-VEGF therapy is not recommended for the treatment of pancreatic cancer, and certain studies have failed to demonstrate the beneficial effect of combining axitinib (an oral inhibitor of VEGF receptors 1, 2 and 3) with gemcitabine $(39,40)$. Due to the essential contribution of stroma in cancer progression, CAFs have recently gained interest as a novel therapeutic target. CAFs are genetically stable, as compared with cancer cells, and thus are more likely to maintain sensitivity to drugs (41). In addition, CAFs are responsible for the structure of tumor ECM, which interferes with the diffusion of anti-cancer agents into tumors (41). Furthermore, communication between CAFs and tumor cells promotes the survival, proliferation and invasiveness of cancer cells. Tumor stroma-directed therapies can target the growth factors and cytokines that initiate the communication within the tumor microenvironment, therefore promoting an anti-cancer effect (41). FAP has previously been suggested as a potential candidate for specifically targeting CAFs (42). Notably, CAF-directed therapy may also be used to overcome the loss of VEGF inhibitor effects. A previous study has revealed that platelet derived growth factor-C (PDGF-C) produced by CAFs is able to initiate VEGF production in tumor cells, thereby sustaining the angiogenic shift. Therefore, antibodies targeting PDGF-C may be used to inhibit angiogenesis in tumors, refractory to anti-VEGF treatments (43).

In conclusion, the present study used CLSM to quantify the expression levels of FAP in a series of human pancreatic adenocarcinomas. An immumohistochemical investigation was also used to determine VEGF expression. A significant correlation was observed between the two markers, and they were both shown to possess prognostic significance. A multivariate analysis demonstrated the prognostic superiority of FAP over VEGF, which is currently considered to be the most consistently reproducible molecular marker with prognostic value in resected pancreatic adenocarcinoma. Since the available systemic therapies for pancreatic adenocarcinoma that target tumor cells have limited clinical benefit, targeting FAP may be a potential novel therapeutic strategy against cancer. However, this is concept that requires further investigation.

\section{References}

1. Siegel R, Naishadham D and Jemal A: Cancer statistics, 2013. CA Cancer J Clinic 63: 11-30, 2013.

2. Crist DW and Cameron JL: The current status of the Whipple operation for periampullary carcinoma. Adv Surg 25: 21-49, 1992.

3. Sohn TA, Yeo CJ, Cameron JL, et al: Resected adenocarcinoma of the pancreas-616 patients: results, outcomes and prognostic indicators. J Gastrointest Surg 4: 567-579, 2000.

4. Cleary SP, Gryfe R, Guindi M, et al: Prognostic factors in resected pancreatic adenocarcinoma: analysis of actual 5-year survivors. J Am Coll Surg 198: 722-731, 2004.

5. Han SS, Jang JY, Kim SW, Kim WH, Lee KU and Park YH: Analysis of long-term survivors after surgical resection for pancreatic cancer. Pancreas 32: 271-275, 2006.

6. Moon HJ, An JY, Heo JS, Choi SH, Joh JW and Kim YI: Predicting survival after surgical resection for pancreatic ductal adenocarcinoma. Pancreas 32: 37-43, 2006.

7. Matsuno S, Egawa S, Fukuyama S, et al: Pancreatic cancer registry in Japan: 20 years of experience. Pancreas 28: 219-230, 2004.

8. Whitehurst B, Flister MJ, Bagaitkar J, et al: Anti-VEGF-A therapy reduces lymphatic vessel density and expression of VEGFR-3 in an orthotopic breast tumor model. Int J Cancer 121: 2181-2191, 2007.

9. Christiansen A and Detmar M: Lymphangiogenesis and cancer. Genes Cancer 2: 1146-1158, 2011.

10. Shinkaruk S, Bayle M, Laïn G and Déléris G: Vascular endothelial cell growth factor (VEGF), an emerging target for cancer chemotherapy. Curr Med Chem Anticancer Agents 3: 95-117, 2003.

11. Brown LF, Guidi AJ, Schnitt SJ, et al: Vascular stroma formation in carcinoma in situ, invasive carcinoma and metastatic carcinoma of the breast. Clin Cancer Res 5: 1041-1056, 1999.

12. Fukumura D, Xavier R, Sugiura T, et al: Tumor induction of VEGF promoter activity in stromal cells. Cell 94: 715-725, 1998.

13. Senger DR, Galli SJ, Dvorak AM, Perruzzi CA, Harvey VS and Dvorak HF: Tumor cells secrete a vascular permeability factor that promotes accumulation of ascites fluid. Science 219: 983-985, 1983.

14. Dvorak HF, Sioussat TM, Brown LF, et al: Distribution of vascular permeability factor (vascular endothelial growth factor) in tumors: concentration in tumor blood vessels. J Exp Med 174: $1275-1278,1991$.

15. Feng D, Nagy JA, Brekken RA, et al: Ultrastructural localization of the vascular permeability factor/vascular endothelial growth factor (VPF/VEGF) receptor-2 (FLK-1, KDR) in normal mouse kidney and in the hyperpermeable vessels induced by VPF/VEGF-expressing tumors and adenoviral vectors. J Histochem Cytochem 48: 545-556, 2000.

16. Leung DW, Cachianes G, Kuang WJ, Goeddel DV and Ferrara N: Vascular endothelial growth factor is a secreted angiogenic mitogen. Science 246: 1306-1309, 1989.

17. Mahadevan D and Von Hoff DD: Tumor-stroma interactions in pancreatic ductal adenocarcinoma. Mol Cancer Ther 6: 1186-1197, 2007.

18. Ikeda N, Adachi M, Taki T, et al: Prognostic significance of angiogenesis in human pancreatic cancer. Br J Cancer 79: 1553-1563, 1999.

19. Itakura J, Ishiwata T, Shen B, Kornmann M and Korc M: Concomitant over-expression of vascular endothelial growth factor and its receptors in pancreatic cancer. Int J Cancer 85: 27-34, 2000. 
20. Fujimoto K, Hosotani R, Wada M, et al: Expression of two angiogenic factors, vascular endothelial growth factor and platelet-derived endothelial cell growth factor in human pancreatic cancer and its relationship to angiogenesis. Eur J Cancer 34: 1439-1447, 1998.

21. Park JE, Lenter MC, Zimmermann RN, Garin-Chesa P, Old LJ and Rettig WJ: Fibroblast activation protein, a dual specificity serine protease expressed in reactive human tumor stromal fibroblasts. J Biol Chem 274: 36505-36512, 1999.

22. Garin-Chesa P, Old LJ and Rettig WJ: Cell surface glycoprotein of reactive stromal fibroblasts as a potential antibody target in human epithelial cancers. Proc Nat Acad Sci USA 87: 7235-7239, 1990.

23. Scanlan MJ, Raj BK, Calvo B, et al: Molecular cloning of fibroblast activation protein alpha, a member of the serine protease family selectively expressed in stromal fibroblasts of epithelial cancers. Proc Nat Acad Sci USA 91: 5657-5661, 1994.

24. Mueller MM and Fusenig NE: Tumor-stroma interactions directing phenotype and progression of epithelial skin tumor cells. Differentiation 70: 486-497 2002.

25. Liotta LA, Steeg PS and Stetler-Stevenson WG: Cancer metastasis and angiogenesis: an imbalance of positive and negative regulation. Cell 64: 327-336, 1991.

26. Cohen SJ, Alpaugh RK, Palazzo I, et al: Fibroblast activation protein and its relationship to clinical outcome in pancreatic adenocarcinoma. Pancreas 37: 154-158, 2008.

27. Goscinski MA, Suo Z, Florenes VA, Vlatkovic L, Nesland JM and Giercksky KE: FAP-alpha and uPA show different expression patterns in premalignant and malignant esophageal lesions. Ultrastruct Pathol 32: 89-96, 2008.

28. Henry LR, Lee HO, Lee JS, et al: Clinical implications of fibroblast activation protein in patients with colon cancer. Clin Cancer Res 13: 1736-1741, 2007.

29. Földes-Papp Z, Demel U and Tilz GP: Laser scanning confocal fluorescence microscopy: an overview. Int Immunopharmacol 3: $1715-1729,2003$

30. Amos WB andWhite JG: How the confocal laser scanning microscope entered biological research. Biol Cell 95: 335-342, 2003.

31. Papaxoinis K, Patsouris E, Kittas C and Nicolopoulou-Stamati P: Insulinlike growth factor I receptor and estrogen receptor beta expressions are inversely correlated in colorectal neoplasms and affected by the insulin resistance syndrome. Hum Pathol 38: 1037-1046, 2007
32. Mason DY, Micklem K and Jones M: Double immunofluorescence labelling of routinely processed paraffin sections. J Pathol 191:452-461, 2000.

33. Hamilton SR and Aaltonen LA: Pathology and Genetics of Tumours of the Digestive System. In: World Health Organization Classification of Tumours. Kleihues P and Sobin LH (eds): IARC Press, Lyon, pp1-250, 2000

34. Giulianotti PC, Boggi U, Fornaciari G, et al: Prognostic value of histological grading in ductal adenocarcinoma of the pancreas. Klöppel vs TNM grading. Int J Pancreatol 17: 279-289, 1995

35. Edge SB, Byrd DR, Compton CC, et al (eds): AJCC Cancer Staging Manual. 7th edition. Springer, New York, NY, pp241-249, 2010.

36. Kraman M, Bambrough PJ, Arnold JN, et al: Suppression of antitumor immunity by stromal cells expressing fibroblast activation protein-alpha. Science 330: 827-830, 2010.

37. Shi M, Yu DH, Chen Y, et al: Expression of fibroblast activation protein in human pancreatic adenocarcinoma and its clinicopathological significance. World J Gastroenterol 18: 840-846, 2012.

38. Smith RA, Tang J, Tudur-Smith C, Neoptolemos JP and Ghaneh P: Meta-analysis of immunohistochemical prognostic markers in resected pancreatic cancer. Br J Cancer 104: 1440-1451, 2011

39. Kindler HL, Ioka T, Richel DJ, et al: Axitinib plus gemcitabine versus placebo plus gemcitabine in patients with advanced pancreatic adenocarcinoma: a double-blind randomised phase 3 study. Lancet Oncol 12: 256-262, 2011.

40. Spano JP, Chodkiewicz C, Maurel J, et al: Efficacy of gemcitabine plus axitinib compared with gemcitabine alone in patients with advanced pancreatic cancer: an open-label randomised phase II study. Lancet 371: 2101-2108, 2008.

41. Cirri P and Chiarugi P: Cancer associated fibroblasts: the dark side of the coin. Am J Cancer Res 1: 482-497, 2011.

42. Rettig WJ, Garin-Chesa P, Healey JH, et al: Regulation and heteromeric structure of the fibroblast activation protein in normal and transformed cells of mesenchymal and neuroectodermal origin. Cancer Res 53: 3327-3335, 1993.

43. Crawford Y, Kasman I, Yu L, et al: PDGF-C mediates the angiogenic and tumorigenic properties of fibroblasts associated with tumors refractory to anti-VEGF treatment. Cancer Cell 15: 21-34, 2009. 